\title{
Subthreshold Pan-Retinal Photocoagulation Using Endpoint Management Algorithm for Severe Nonproliferative Diabetic Retinopathy: A Paired Controlled Pilot Prospective Study
}

\author{
Kunbei Lai Hongkun Zhao Lijun Zhou Chuangxin Huang Xiaojing Zhong \\ Yajun Gong Longhui Li Fabao Xu Cong Li Lin Lu Chenjin Jin \\ State Key Laboratory of Ophthalmology, Zhongshan Ophthalmic Center, Sun Yat-sen University, Guangzhou, China
}

\section{Keywords}

Subthreshold photocoagulation · Endpoint management ·

Pattern scan laser · Severe nonproliferative

diabetic retinopathy $\cdot$ Pan-retinal photocoagulation

\begin{abstract}
Purpose: The aim of this study was to report the efficacy and safety profile of subthreshold pan-retinal photocoagulation (PRP) using endpoint management (EPM) algorithm compared with conventional threshold PASCAL PRP for the treatment of severe nonproliferative diabetic retinopathy (NPDR). Methods: This was a prospective, single-center, paired randomized controlled trial of 56 eyes of 28 participants with bilateral symmetric severe NPDR. One eye of the participant was randomly assigned to receive the subthreshold EPM $\mathrm{PRP}$, while the other eye of the same participant received the threshold PASCAL PRP. The primary outcome measures included the difference in the 1-year risk of progression to PDR between 2 groups, and mean changes of the logarithm of the minimal angle of resolution (logMAR) visual acuity (VA). The second outcome measures included central foveal thickness (CFT), 1-year risk of progression to PDR, and visual field (VF) parameters. Results: The subthreshold EPM PRP group and the threshold PASCAL PRP group had similar 1-year risk of progression to PDR during the 12-month follow-up visits
\end{abstract}

karger@karger.com

(c) 2020 S. Karger AG, Basel

www.karger.com/ore

Karger!
(17.86 vs. $14.29 \%, p>0.05)$. Slightly decreased VA was found in both groups (0.08 vs. 0.09 logMAR VA); however, no statistical difference was found for neither group $(p>0.05)$. Similar results were found for thickened CFT for both groups (23.59 vs. $28.34 \mu \mathrm{m}, p>0.05)$. Specifically, although substantial loss of VF was found in the threshold PASCAL PRP group ( $p<$ 0.05), no obvious damage to VF was seen in the subthreshold EPM PRP group ( $p>0.05$ ). Conclusion: The subthreshold EPM PRP is noninferior to the conventional threshold PASCAL PRP in the treatment of severe NPDR during 12-month follow-up and could be an alternative treatment option for patients with severe NPDR.

๑) 2020 S. Karger AG, Basel

\section{Introduction}

Diabetic retinopathy is the leading cause of visual impairment in working-age adults worldwide [1, 2]. Diabetic retinopathy is clinically classified into 2 types based on the development of retinal neovascularization, namely, nonproliferative diabetic retinopathy (NPDR) and proliferative diabetic retinopathy (PDR) [3]. The Early

Kunbei Lai and Hongkun Zhao contributed equally to this work.
Correspondence to:

Chenjin Jin, jinchj@ mail.sysu.edu.cn 
Treatment Diabetic Retinopathy Study (ETDRS) demonstrated that visible endpoint pan-retinal photocoagulation (PRP) by laser treatment remained the standard intervention for patients with severe NPDR and PDR $[4,5]$. However, treatment with PRP has complications and side effects including vision loss due to causation or exacerbation of macular edema, peripheral visual field (VF) defects, night-vision loss, reduction in color and contrast sensitivity, and fibrovascular traction [6]. Therefore, many attempts including parameters of laser treatment protocol and new laser instruments are being made to minimize these complications and side effects [7].

Pattern scan laser (PASCAL ${ }^{\circledR}$ ), first described by Blumenkranz et al. [8], is a semiautomated laser retinal photocoagulator allowing rapid application of multiple laser spots on the retina with a shorter pulse duration. Laser photocoagulation of the retina using PASCAL with a short pulse duration of $20 \mathrm{~ms}$ has been demonstrated to possess similar efficiency to that of conventional retinal photocoagulation with a long pulse duration of $200 \mathrm{~ms}$ for the treatment of severe NPDR or PDR [9]. At the same time, since PRP with PASCAL delivered less severe threshold burns than with conventional laser, better toleration and less side effects were found for PASCAL with a short pulse duration [10-12].

In order to further reduce the laser-induced retinal damage, an advance algorithm called "end point management (EPM)" was created based on PASCAL laser, which was able to produce a subvisible laser lesion [13]. To date, only 2 small case series researches have been published that subthreshold photocoagulation with EPM is effective for the treatment of diabetic macular edema (DME) and central serous chorioretinopathy $[14,15]$. However, no data of subthreshold photocoagulation using EPM algorithm for treating diabetic retinopathy has been published. Herein, we presented for the first time the efficacy and safety profiles of the subthreshold EPM PRP compared with the threshold PASCAL PRP for the treatment of severe NPDR.

\section{Materials and Methods}

This was a prospective, single-center, paired randomized controlled trial conducted on 56 eyes of 28 patients with a diagnosis of naive, bilateral, symmetric, severe NPDR who presented to Zhongshan Ophthalmic Center, Sun Yat-sen University, Guangzhou, China, in the period from March 29, 2016, to January 10, 2020. This study was approved by the Institutional Review Board of Sun Yat-sen University (2016MEKY004), and all procedures were conducted in accordance with the tenets of the Declaration of Helsinki. Written informed consent was obtained from all subjects after explanation of the nature and possible consequences of the study. This study was registered on the https://www.clinicaltrials. gov (trial registration number: NCT01759121).

\section{Study Population}

Study participants were at least 18 years old with type 1or type 2 diabetes. The inclusion criteria were 1) patients were diagnosed with bilateral, symmetric, severe NPDR. The diagnosis of severe NPDR was made on the basis of the Diabetic Retinopathy Study Research Group [16]; 2) best corrected visual acuity (BCVA) was $20 / 100$ or better on Snellen chart; 3 ) no previous treatment such as PRP or antivascular endothelial growth factor (VEGF) treatment; and 4) serum glycosylated hemoglobin $(\mathrm{HbAlc})<10 \%$. Exclusion criteria included 1) any other ocular disease, such as ocular trauma, glaucoma, uveitis, angioid streaks, or pathologic myopia; 2) media opacities such as corneal opacity, significant cataract, or vitreous hemorrhage obscuring fundus examination; and (3) any severe uncontrolled systemic disease, such as uncontrolled hypertension (blood pressure over 140/90 $\mathrm{mm} \mathrm{Hg}$ should be excluded in this study because hypertension had been demonstrated to be positively associated with prevalence and progression of diabetic retinopathy), coronary heart disease, liver failure, kidney failure (who was not suitable for the examination of fundus fluorescein angiography [FFA] depended on the assessment and judgment of kidney specialists or liver specialists), or mental disease (who was not cooperative with examinations such as FFA and optical coherence tomography $[\mathrm{OCT}]$ and treatment of laser photocoagulation).

\section{Study Design}

Both eyes with symmetric severe NPDR of the same patient were randomized into 2 treatment groups using the computerized randomization procedure with sequentially numbered, opaque, sealed envelopes: 1 eye was randomly assigned to receive the subthreshold EPM PRP, the other eye received the threshold PASCAL PRP. The treatment allocation envelopes remained sealed until the interventions were assigned. The primary outcome follow-up visit was at 1 years, with follow-up visits on months 1, 3, 6, 9, and 12 .

\section{Treatment Protocol}

Performance of threshold PASCAL PRP: Threshold laser photocoagulation of the retina was performed after local anesthesia using a laser PASCAL photocoagulator (Topcon Medical laser systems Inc., Livermore, CA, USA). The single spot laser with $30 \mathrm{~ms}$ individual impulse duration and $200 \mu \mathrm{m}$ spot size was used for titration: the laser power was gradually increased until a gray white laser burn on retina was achieved. Then pattern of $3 \times 3-5 \times 5$ was used for PRP in 2 sessions within 7 days.

Performance of subthreshold PRP using EPM algorithm: The Endpoint Management ${ }^{\mathrm{TM}}$ with Landmark Pattern ${ }^{\mathrm{TM}}$ was applied using the same laser pascal photocoagulator for the subthreshold PRP in this study. EPM utilizes advanced algorithms to control both power and exposure time of the laser, and Landmark Patterns provides the periphery "landmarking" of the applied pattern by setting the 4 corners of the pattern to the $100 \%$ level (barely visible level). Briefly, titration was performed in the same way as threshold PRP to obtain barely visible burn, then irradiation with pattern of $3 \times 3-5 \times 5$ was conducted after switching over to EPM mode of $50 \%$ setting.

If there was clinically significant macular edema at the initial visit, focal or grid photocoagulation would be applied at the same 
day of PRP with 20 ms exposure time, 100- $\mu \mathrm{m}$ spot size, and appropriate laser power to obtain a light gray barely visible laser lesion in the macular area as previously described $[17,18]$. Rescue threshold or threshold PRP was offered if progression of PDR (development of neovascular complexes) was noted. Retreatment of focal or grid macular photocoagulation would be applied if there was persistence of macular edema at the follow-up visits. AntiVEGF therapy was always available to all patients if DME was not controlled by focal or grid photocoagulation. But if the patient chose the anti-VEGF therapy, he/she should be excluded from this study according to the protocol of the study since anti-VEGF therapy may prevent the progress of diabetic retinopathy. But we promised that their choices would have no influence on their further treatments and follow-ups.

\section{Ophthalmic Examinations and Follow-Up}

BCVA, noncontact tonometer (NCT), comprehensive slitlamp examination, fundus photographs (7 fields), and OCT (Spectralis ${ }^{\circledR}$ HRA + OCT; Heidelberg Engineering, Germany) were performed at baseline and at each follow-up visits on months 1, 3, 6, 9 , and 12. FFA were carried out at baseline and at months 6 and 12. Humphrey VF testing (Carl Zeiss Meditec, Germany) on 24-2 were performed at baseline and at month 12. Additional follow-up visits and examinations including OCT, FFA, and VF testing would be arranged if the patients had severe vision loss or when the ophthalmologist considered it was necessary.

BCVA was measured using a Snellen chart and converted the values to $\log$ MAR equivalent VA which could be used for statistical analysis directly. The difference in mean changes of logMAR VA was investigated using the one-way repeated-measures analysis of variance. Central foveal thickness (CFT) was determined by averaging the values of vertical and horizontal CFT measured manually from the inner retinal surface to the retinal pigmented epithelium (RPE) line as we previously described [19]. For the analysis of parameters of VF testing, 2 of the global indices including mean deviation (MD) and corrected pattern standard deviation (CPSD) were used [20]. MD provided an overall value of the total amount of VF loss, while CPSD showed any irregularities in the VF irrespective of any overall depression after adjusting for errors of threshold determination [21].

\section{Outcomes}

The primary outcome measures included the difference in the 1 -year risk of progression to PDR between 2 groups and the mean changes of the logarithm of the minimal angle of resolution (logMAR) visual acuity (VA). The second outcome measures included CFT, the difference in the 1-year risk of progression to PDR between 2 groups, and VF parameters including MD and CPSD. And the treatment failure end points of this study were either the development of vitreous hemorrhage or performance of pars plana vitrectomy.

\section{Statistical Analysis}

All data were expressed as the means \pm SE except for special instructions. Data were analyzed using SPSS 19.0 software (IBM Corporation, Armonk, NY, USA). BCVA was measured using a Snellen chart and was converted the values to logMAR equivalent VA which could be used for statistical analysis directly. Baseline parciticants' characteristics and outcomes between study groups were compared using $\chi^{2}$ test for categorical variables and 2-sample $t$ test for continuous variables, and cluster effect within eyes was
Table 1. Baseline demographics and clinical characteristics of all randomized participants

\begin{tabular}{lrrr}
\hline & $\begin{array}{l}\text { Subthreshold } \\
\text { EPM }^{\mathrm{sm}} \\
\text { PRP group }(n=28)\end{array}$ & $\begin{array}{l}\text { Threshold } \\
\text { PASCAL } \\
\text { PRP group } \\
(n=28)\end{array}$ & $p$ value \\
& $57.96(11.33)$ & $57.96(11.33)$ & $1.0^{\dagger}$ \\
\hline $\begin{array}{l}\text { Mean age, years (SD) } \\
\text { Sex, } n(\%)\end{array}$ & $13(46.4)$ & $13(46.4)$ & $1.0^{*}$ \\
$\quad \begin{array}{l}\text { Female } \\
\text { Male }\end{array}$ & $15(53.6)$ & $15(53.6)$ & $1.0^{*}$ \\
$\begin{array}{l}\text { Eye, } n(\%) \\
\text { Right }\end{array}$ & $12(42.9)$ & $16(57.1)$ & $0.285^{*}$ \\
Left & $16(57.1)$ & $12(42.9)$ & $0.285^{*}$ \\
BCVA (logMAR) & $0.16 \pm 0.03$ & $0.15 \pm 0.04$ & $0.899^{\dagger}$ \\
$\quad \begin{array}{l}\text { Mean (SE) } \\
\text { Range }\end{array}$ & $0-0.4$ & $-0.1-0.5$ & \\
CFT, mean (SE), $\mu \mathrm{m}$ & $271.00 \pm 13.29$ & $293.07 \pm 16.73$ & $0.453^{\dagger}$ \\
\end{tabular}

EPM, end point management; PRP, panretinal photocoagulation; PASCAL, pattern scan laser; SD, standardized deviation; SE, standard error; BCVA: best-corrected visual acuity; LogMAR, logarithm of the minimum angle of resolution; CFT, central foveal thickness. ${ }^{*}$ Pearson $\chi^{2}$ test. ${ }^{\dagger}$ Two-sample $T$ test.

taken into account. Each of the follow-up visits was compared with baseline in mean logMAR VA and CFT by study group separately using paired $t$ test. The difference of change since baseline in outcomes between study groups was tested by 2 -sample $t$ test adjusting for correlation between 2 eyes. A 2 -sided $p$ value $<0.05$ was considered statistically significant.

\section{Results}

Completed data of 56 eyes of 28 participants between March 29, 2016, and January 10, 2020, were obtained. Twenty-eight eyes were assigned randomly to the subthreshold EPM PRP group, and other 28 eyes were assigned to the threshold PASCAL PRP group (shown in Fig. 1). The median ages were 58.5 years (interquartile range [IQR], $47-69$ years) and $46.43 \%$ were women. The mean $\operatorname{logMAR} V A$ was $0.16 \pm 0.03$ and $0.15 \pm 0.04$ in the subthreshold EPM PRP group and the threshold PASCAL PRP group, respectively. DME at baseline was present in 5 patients (17.86\%) the subthreshold EPM PRP group and in 6 patients $(21.43 \%)$ in threshold PASCAL PRP group, respectively. As was shown in Table 1, the baseline demographic and clinical characteristics of the patients in 2 groups appeared similar. Parameters for subthreshold EPM PRP and threshold PASCAL PRP were shown in Table 2. 
Fig. 1. Flowchart for the study sample.



\section{Best Corrected Visual Acuity}

The mean logMAR VA at baseline in both the groups was comparable, with a value of $0.16 \pm 0.03$ in the subthreshold EPM PRP group and $0.15 \pm 0.04$ in the threshold PASCAL PRP group $(p>0.05)$. The VA dropped to $0.19 \pm 0.03,0.19 \pm 0.03,0.20 \pm 0.04,0.22 \pm$ $0.04,0.24 \pm 0.04$ in the subthreshold EPM PRP group, while the VA dropped to $0.20 \pm 0.03,0.21 \pm 0.04,0.21$ $\pm 0.03,0.21 \pm 0.04,0.24 \pm 0.03$ in the threshold PASCAL PRP group at the follow-ups of $1,3,6,9$, and 12 months. However, no statistical difference was found at any follow-up visit compared with baseline in both groups $(p>0.05)$ (shown in Fig. 2a). Specifically, the drop of $0.08 \log$ MAR VA (approximate equivalent 4 letters on the ETDRS chart) in the subthreshold EPM PRP group versus 0.09 logMAR VA (approximate equivalent 4.5 letters on the ETDRS chart) in the threshold PASCAL PRP group was found to be insignificant at month $12(p>0.05)$.

\section{Central Foveal Thickness}

The CFT in the subthreshold EPM PRP group were $271.00 \pm 13.29 \mu \mathrm{m}, 292.62 \pm 19.43 \mu \mathrm{m}, 295.16 \pm 17.93 \mu \mathrm{m}$, $304.71 \pm 20.59 \mu \mathrm{m}, 307.51 \pm 16.74 \mu \mathrm{m}$, and $294.59 \pm 16.46$ $\mu \mathrm{m}$ at baseline and at the follow-ups of $1,3,6,9$, and 12 months, respectively. Similarly, the CFT in the threshold
PASCAL PRP group was $293.07 \pm 16.73 \mu \mathrm{m}$ at baseline, which increased to $326.41 \pm 21.39 \mu \mathrm{m}, 328.23 \pm 25.10 \mu \mathrm{m}$, $339.88 \pm 25.64 \mu \mathrm{m}, 325.44 \pm 24.17 \mu \mathrm{m}$, and $321.41 \pm 22.19$ $\mu \mathrm{m}$ at the follow-ups of $1,3,6,9$, and 12 months. However, there was no statistical difference for the CFT at any follow-up compared with that of baseline in both groups $(p>0.05)$ (shown in Fig. 2b). Specifically, the increased $23.59 \mu \mathrm{m}$ of CFT from baseline to month 12 in the subthreshold EPM PRP group versus $28.34 \mu \mathrm{m}$ in the threshold PASCAL PRP group was found to be insignificant $(p>0.05)$.

\section{Disease Progression}

During the 12-month follow-up, 5 eyes (17.86\%) developed to PDR including 4 eyes demonstrated neovascularization and 1 eye demonstrated vitreous hemorrhage in the subthreshold EPM PRP group. Specifically, since the patient had sustained vitreous hemorrhage more than 1 month, he finally received the pars plana vitrectomy surgery. In the threshold PASCAL PRP group, there were 4 eyes (14.29\%) developed to PDR, all of which were demonstrated neovascularization during the 12-month follow-up. The percentage rate of progression to PDR during the 12 months had no statistical difference between 2 groups $(p>0.05)$. Moreover, a total of 7 times of rescue PRP were performed in the subthreshold EPM PRP group 


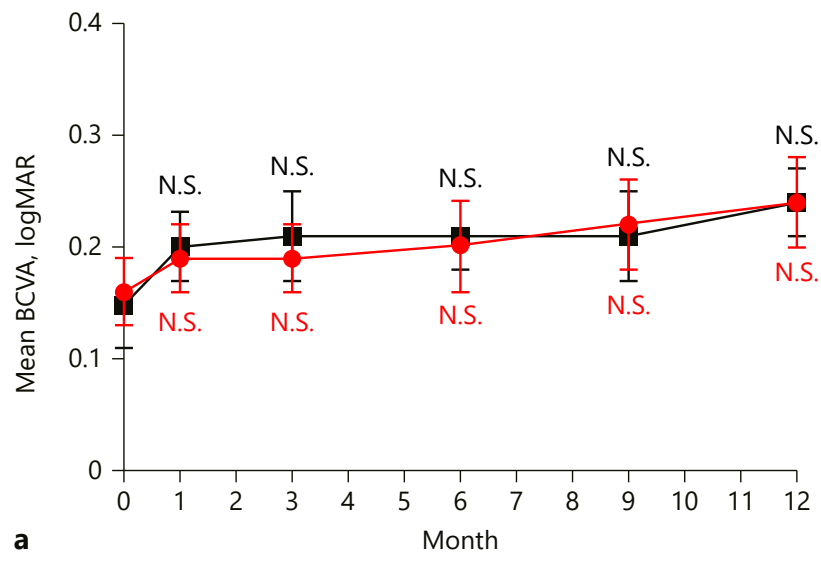

Fig. 2. Time courses of the best-corrected visual acuity (BCVA) in logarithm of the minimum angle of resolution (logMAR) visual acuity (VA) and CFT during the 12-month follow-up. a Although slightly decreased VA were found in both groups, no statistical difference was found at any follow-up visit compared with baseline in the subthreshold EPM PRP group or the threshold PASCAL

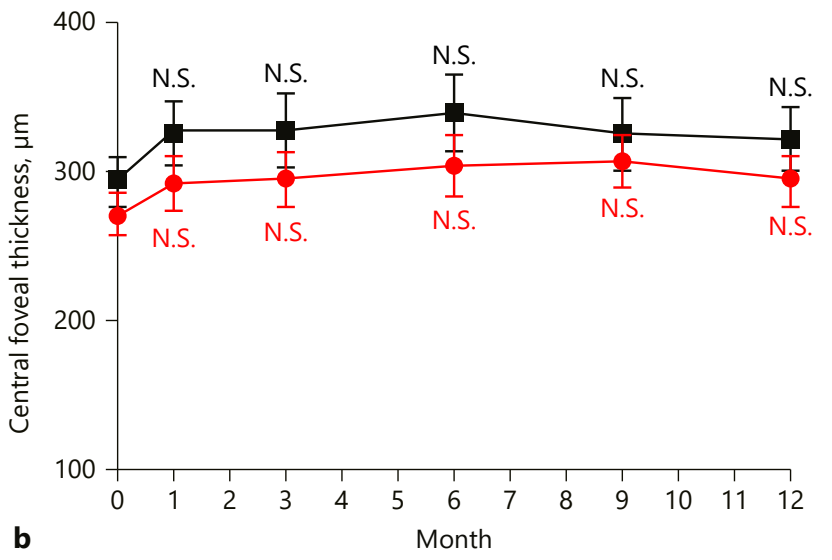

PRP group (all $p>0.05$ ). b Besides, there was no statistical difference for the changes of $\log M A R$ VA between the subthreshold EPM PRP group and the threshold PASCAL PRP group at any follow-up visit (all $p>0.05)$. Similar results were found for the CFT for both groups. No statistical difference was found for the increased CFT between 2 groups at any follow-up visit (all $p>0.05$ ).

Table 2. Laser parameters of subthreshold EPM PRP and threshold PASCAL PRP

\begin{tabular}{|c|c|c|}
\hline & Subthreshold $\mathrm{EPM}^{\mathrm{TM}} \mathrm{PRP}$ & Threshold PASCAL ${ }^{\circledR}$ PRP \\
\hline Wavelength, nm & 532 & 532 \\
\hline Pulse duration, ms & 30 & 30 \\
\hline Pattern and spacing & $3 \times 3-5 \times 5$ with 1 spot spacing & $3 \times 3-5 \times 5$ with 1 spot spacing \\
\hline Total no. of laser spots (mean \pm SD) & $3,623.2 \pm 438.9$ & $3,625.1 \pm 421.9$ \\
\hline Treatment times & Two sessions & Two sessions \\
\hline
\end{tabular}

EPM, end point management; PRP, pan-retinal photocoagulation; PASCAL, pattern scan laser; SD, standardized deviation.

because of the development of active neovascularization. In contrast, 6 times of rescue PRP were performed in the threshold PASCAL PRP group.

\section{Visual Field Parameters}

The average MD increased from $-8.17 \pm 1.33 \mathrm{~dB}$ at baseline to $-10.45 \pm 1.09 \mathrm{~dB}$ at month 12 in the subthreshold EPM PRP group; however, no statistical difference was found between month 12 and baseline $(p>0.05)$.
While the average MD increased from $-6.45 \pm 1.06 \mathrm{~dB}$ at baseline to $-11.66 \pm 1.19 \mathrm{~dB}$ with statistical difference in the threshold PASCAL PRP group $(p<0.05)$ (shown in Fig. 3a).

Similar results were found for CPSD, the CPSD value increased from $4.50 \pm 0.46 \mathrm{~dB}$ at the baseline to $5.19 \pm 0.44$ $\mathrm{dB}$ at month 12 with no statistical difference in the subthreshold EPM PRP group ( $p>0.05$ ), while the value of CPSD increased from $3.62 \pm 0.36 \mathrm{~dB}$ at the baseline to 


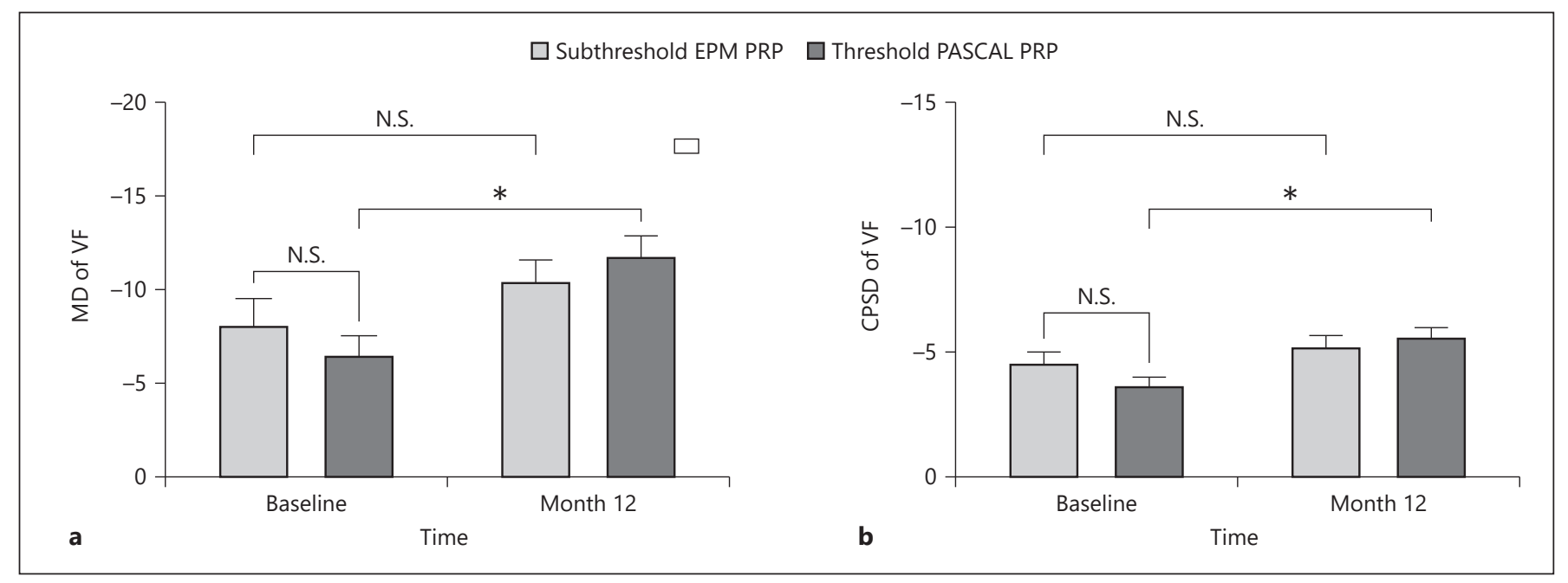

Fig. 3. Influences of subthreshold laser pan-retinal photocoagulation (PRP) with endpoint management (EPM) algorithm and the threshold PASCAL PRP on parameters of visual field (VF). There was no statistical difference for neither mean deviation (MD) (a, the first bar vs. the second bar) nor corrected pattern standard deviation (CPSD) (b, the first bar vs. the second bar) at baseline between 2 groups $(p>0.05) .12$ months after laser photocoagulation, no statistical difference was found for neither the MD values $(-10.45 \pm 1.09 \mathrm{~dB}$ at month 12 vs. $-8.17 \pm 1.33 \mathrm{~dB}$ at baseline, $p>$

$5.56 \pm 0.39 \mathrm{~dB}$ at month 12 with statistical difference in the threshold PASCAL PRP group $(p<0.05)$ (shown in Fig. 3b).

\section{Safety Profiles}

Aggravation of cataract occurred in 2 patients ( 1 in the subthreshold EPM PRP group and the other in the threshold PASCAL PRP group). One patient died during the follow-up due to myocardial infraction, which had been confirmed to be with no relationship with PRP. Intraocular pressure was stable in all eyes throughout the study. No other ocular or systemic complications were observed.

\section{Discussion}

Diabetic retinopathy is the leading cause of visual impairment in working-age adults worldwide $[1,2]$. Diabetic Retinopathy Study (DRS) and ETDRS have confirmed that PRP decreases the risk of severe vision loss by $>50 \%$ for patients with PDR $[4,22]$; however, the best timing to initiate PRP for which stage of diabetic retinopathy is still being under heated debate [23]. The ET-
0.05) (a, the third bar vs. the first bar) nor the CPSD values (5.19 $\pm 0.44 \mathrm{~dB}$ at month 12 vs. $4.50 \pm 0.46 \mathrm{~dB}$ at baseline, $p>0.05)(\mathbf{b}$, the third bar vs. the first bar) in the subthreshold EPM PRP group compared with those of baseline. However, statistical difference was found for both MD values $(-11.66 \pm 1.19 \mathrm{~dB}$ at month vs. $-6.45 \pm 1.06 \mathrm{~dB}$ at baseline, $p<0.05)(\mathbf{a}$, the fourth bar vs. the second bar) and CPSD $(5.56 \pm 0.39 \mathrm{~dB}$ at month 12 vs. $3.62 \pm 0.36 \mathrm{~dB}$ at the baseline, $p<0.05)$ (b, the fourth bar vs. the second bar) values in threshold PASCAL PRP group.
DRS, published in 1991, was the only trial designed to determine the best time to initiate PRP, and the data showed that early PRP for patients with NPDR or early PDR reduced the risk of severe visual loss after 5 years by 23\% compared with deferred PRP, although this study did not provide results separately for NPDR and early PDR [24]. However, considering the inherent retinal damage in conventional photocoagulation [25-30], many attempts including parameters of laser protocol as well as new laser instruments are being made to minimize complications and side effects of conventional photocoagulation [7]. Luttrull and coworkers [31] reported in a retrospective study that subthreshold diode micropulse PRP minimized retinal damage and side effects in the treatment of high-risk NPDR and PDR [31]. More recently, Luttrull et al. [31] reported that subthreshold microsecond PRP is noninferior to continuous-wave PASCAL PRP in a small randomized pilot study including 20 eyes of 10 subjects ( 4 eyes with NPDR and 16 eyes with PDR).

Actually, up to date, no evidence has been presented that the retinal destruction inherent in visible end point photocoagulation is actually necessary to achieve a therapeutic effect for severe NPDR [7, 31, 32]. With this in 
mind, we describe the subthreshold PRP using advanced algorithm called "EPM" in the management of severe NPDR. EPM is a computational model-based algorithm for precisely control and optimize laser power and duration to achieve subthreshold laser treatment to the eyes in order to eliminate side effects while at the same time maintaining clinical efficacy [13]. Our data showed that the subthreshold EPM PRP group and the threshold PASCAL PRP group had similar 1-year risk of progression to PDR during the 12-month follow-up visits (17.86 vs. $14.29 \%, p>0.05)$. Compared with the progression rate of $52 \%$ for untreated patients with severe NPDR reported by ETDRS [33], our data showed that both laser treatments showed efficacy for the treatment of severe NPDR. Although slightly decreased VA was found in both groups, no statistical difference was found at any follow-up visit compared with baseline in both groups, suggesting that VA remained stable during the 12-month follow-up in both groups (shown in Fig. 2a). Our data also showed that subthreshold EPM PRP induced less thickened CFT compared with the threshold PASCAL PRP group, although no statistical difference was found between 2 groups ( 23.59 vs. $28.34 \mu \mathrm{m}, p>0.05$ ) (shown in Fig. 2b). Moreover, although substantial loss of VF was found to be associated with application of the threshold PASCAL PRP 12 months after laser photocoagulation $(p<0.05)$ (shown in Fig. 3a, b), no obvious damage to VF was seen in the subthreshold EPM PRP group 12 months after laser photocoagulation $(p>0.05)$ (shown in Fig. 3a, b).

Interestingly, a recent in vitro study revealed that subthreshold diode micropulse laser treatment could suppress the expression of VEGF in the RPE cells without damaging the RPE cells [34]. Since VEGF is the critical neovasculization-promoting cytokine in the development of PDR, we speculated it that the treatment effects of subthreshold EPM PRP for severe NPDR might partially due to the biological modulation of RPE cells. However, the exact detailed mechanisms are warranted in future study.

Moreover, excepting for less damage to retina and less side effects, subthreshold EPM PRP has other advantages. In our study, subthreshold EPM PRP was performed with the technology called Landmark Patterns ${ }^{\mathrm{TM}}$, which allowed the physician well control and visibility of laser delivery by simply setting the desired endpoint and tracking it during the treatment. Besides, all the patients in our study have good acceptance of the performance of subthreshold EPM PRP without complaint of painful feeling.
Limitations of our study included a small sample size and short follow-up. According to our previous calculation of sample size, 84 patients should be enrolled in the study to present the statistical difference for mean changes of the logMAR VA, therefore, more patients and longer follow-up period would be needed in future study. In addition, owing to lack of other retinal functional data like electroretinography (ERG) and contrast sensitivity, we are unable to comment more detailed influence of subthreshold EPM PRP on retinal function. However, strength of our study included 2 laser treatment modalities in 2 eyes of the same patient with symmetric stages of severe NPDR, which avoided the selection bias. Together with the prospective, paired randomized controlled design of this study, we believe that subthreshold EPM PRP is noninferior to conventional threshold PASCAL PRP for the treatment of severe NPDR at least for 1-year follow-up. Even so, larger, long-term, multicenter, double-blind, and prospective clinical studies are still necessary to confirm the longterm efficacy and safety of subthreshold EPM PRP in the treatment of severe NPDR.

In conclusion, our prospective study proposes that subthreshold EPM PRP is noninferior to conventional threshold PASCAL PRP in the treatment of severe NPDR. Subthreshold EPM PRP could be an alternative treatment option for patients with severe NPDR, which minimizes the damage to retina and reduces complications and side effects of laser photocoagulation.

\section{Statement of Ethics}

This study was approved by the Institutional Review Board of Sun Yat-sen University (2016MEKY004), and all procedures were conducted in accordance with the tenets of the Declaration of Helsinki. Written informed consent was obtained from all subjects after explanation of the nature and possible consequences of the study.

\section{Conflict of Interest Statement}

The authors have no conflicts of interest to declare.

\section{Funding Sources}

This study was supported by grants from Project 5010 of Clinical Research of Sun Yat-sen University (2013007), Key Projects of Guangzhou Science and Technology Plan (201707020008), Fundamental Research Funds for the Central Universities (Sun Yatsen University, 19ykpy152), the Natural Science Foundation of 
Guangdong Province (2020A1515011099), and Basic Research Plan of Guangzhou Science and Technology Plan Project (202002030380). The funding sponsors had no roles in the design of the study and collection, analysis, and interpretation of data as well as in writing the manuscript.

\section{Author Contributions}

K.L. and H.Z. contributed to the data analysis and drafted the paper. L.Z., C.H., X.Z., Y.G., L.L., F.X., C.L., and L.L. made contributions to acquisition of data and drafting. C.J. designed of the study and was responsible for revising the paper. All authors have read and approved the manuscript.

\section{References}

1 Klein R, Lee KE, Knudtson MD, Gangnon RE, Klein BE. Changes in visual impairment prevalence by period of diagnosis of diabetes: the Wisconsin epidemiologic study of diabetic retinopathy. Ophthalmology. 2009 Oct;116(10):193742.

2 Shaw JE, Sicree RA, Zimmet PZ. Global estimates of the prevalence of diabetes for 2010 and 2030. Diabetes Res Clin Pract. 2010 Jan;87(1): 4-14.

3 Cunha-Vaz J, Ribeiro L, Lobo C. Phenotypes and biomarkers of diabetic retinopathy. Prog Retin Eye Res. 2014 Jul;41:90-111.

4 Diabetic Retinopathy Study Research Group. Preliminary report on effects of photocoagulation therapy. The diabetic retinopathy study research group. Am J Ophthalmol. 1976 Apr; 81(4):383-96.

5 The Early Treatment Diabetic Retinopathy Study Research Group. Techniques for scatter and local photocoagulation treatment of diabetic retinopathy: early treatment diabetic retinopathy study report no. 3. Int Ophthalmol Clin. 1987;27(4):254-64.

6 Liang JC, Huamonte FU. Reduction of immediate complications after panretinal photocoagulation. Retina. 1984;4(3):166-70.

7 Chhablani J, Roh YJ, Jobling AI, Fletcher EL, Lek JJ, Bansal P, et al. Restorative retinal laser therapy: present state and future directions. Surv Ophthalmol. 2018 May-Jun;63(3):307-28.

8 Blumenkranz MS, Yellachich D, Andersen DE, Wiltberger MW, Mordaunt D, Marcellino GR, et al. Semiautomated patterned scanning laser for retinal photocoagulation. Retina. 2006 Mar; 26(3):370-6.

9 Nemcansky J, Stepanov A, Nemcanska S, Masek P, Langrova H, Studnicka J. Single session of pattern scanning laser versus multiple sessions of conventional laser for panretinal photocoagulation in diabetic retinopathy: efficacy, safety and painfulness. PloS One. 2019;14(7):e0219282.

10 Muqit MM, Marcellino GR, Gray JC, McLauchlan R, Henson DB, Young LB, et al. Pain responses of Pascal $20 \mathrm{~ms}$ multi-spot and $100 \mathrm{~ms}$ singlespot panretinal photocoagulation: Manchester pascal study, MAPASS report 2. Br J Ophthalmol. 2010 Nov;94(11):1493-8.

11 Muqit MM, Sanghvi C, McLauchlan R, Delgado C, Young LB, Charles SJ, et al. Study of clinical applications and safety for Pascal(R) laser photocoagulation in retinal vascular disorders. Acta ophthalmologica. 2012 Mar;90(2):155-61.

12 Muqit MM, Young LB, McKenzie R, John B, Marcellino GR, Henson DB, et al. Pilot ran- domised clinical trial of Pascal TargETEd retinal versus variable fluence PANretinal $20 \mathrm{~ms}$ laser in diabetic retinopathy: peter pan study. Br J Ophthalmol. 2013 Feb;97(2):220-7.

13 Lavinsky D, Sramek C, Wang J, Huie P, Dalal R, Mandel Y, et al. Subvisible retinal laser therapy: titration algorithm and tissue response. Retina. 2014 Jan;34(1):87-97.

14 Lavinsky D, Palanker D. Nondamaging photothermal therapy for the retina: initial clinical experience with chronic central serous retinopathy. Retina. 2015 Feb;35(2):213-22.

15 Hamada M, Ohkoshi K, Inagaki K, Ebihara N, Murakami A. Subthreshold photocoagulation using endpoint management in the PASCAL ${ }^{\circledR}$ system for diffuse diabetic macular edema. J Ophthamol. 2018;2018:7465794.

16 The Diabetic Retinopathy Study Research Group. Photocoagulation treatment of proliferative diabetic retinopathy: the second report of diabetic retinopathy study findings. Ophthalmology. 1978 Jan;85(1):82-106.

17 Bolz M, Kriechbaum K, Simader C, Deak G, Lammer J, Treu C, et al. In vivo retinal morphology after grid laser treatment in diabetic macular edema. Ophthalmology. 2010 Mar;117(3):53844.

18 Jain A, Collen J, Kaines A, Hubschman JP, Schwartz S. Short-duration focal pattern grid macular photocoagulation for diabetic macular edema: four-month outcomes. Retina. 2010 Nov-Dec;30(10):1622-6.

19 Lai K, Li Y, Zhou L, Zhong X, Huang C, Xu F, et al. Comparison of the effects of photodynamic therapy, intravitreal ranibizumab and combination for polypoidal choroidal vasculopathy under 1 + PRN regimen. BMC Ophthalmol. 2018 Jun 20;18(1):144.

20 Pahor D. Visual field loss after argon laser panretinal photocoagulation in diabetic retinopathy: full- versus mild-scatter coagulation. Int Ophthalmol. 1998;22(5):313-9.

21 Thomas R, George R. Interpreting automated perimetry. Indian J Ophthalmol. 2001 Jun;49(2): $125-40$.

22 The Early Treatment Diabetic Retinopathy Study Research Group. Treatment techniques and clinical guidelines for photocoagulation of diabetic macular edema. Early Treatment Diabetic Retinopathy Study Report Number 2. Ophthalmology. 1987 Jul;94(7):761-74.

23 Royle P, Mistry H, Auguste P, Shyangdan D, Freeman K, Lois N, et al. Pan-retinal photocoagulation and other forms of laser treatment and drug therapies for non-proliferative diabetic ret- inopathy: systematic review and economic evaluation. Health Technol Assess. 2015 Jul;19(51): $\mathrm{v}-247$.

24 The Early Treatment Diabetic Retinopathy Study Research Group. Early photocoagulation for diabetic retinopathy. ETDRS report number 9. Ophthalmology. 1991 May;98(5 Suppl l):76685.

25 Photocoagulation treatment of proliferative diabetic retinopathy: relationship of adverse treatment effects to retinopathy severity. Diabetic retinopathy study report no. 5. Developments in ophthalmology. 1981;2:248-61.

26 Higgins KE, Meyers SM, Jaffe MJ, Roy MS, de Monasterio FM. Temporary loss of foveal contrast sensitivity associated with panretinal photocoagulation. Arch Ophthalmol. 1986 Jul; 104(7):997-1003.

27 Lewis H, Schachat AP, Haimann MH, Haller JA, Quinlan P, von Fricken MA, et al. Choroidal neovascularization after laser photocoagulation for diabetic macular edema. Ophthalmology. 1990 Apr;97(4):503-1.

28 Guyer DR, D'Amico DJ, Smith CW. Subretinal fibrosis after laser photocoagulation for diabetic macular edema. Am J Ophthalmol. 1992 Jun 15; 113(6):652-6.

29 Henricsson M, Heijl A. The effect of panretinal laser photocoagulation on visual acuity, visual fields and on subjective visual impairment in preproliferative and early proliferative diabetic retinopathy. Acta Ophthalmol. 1994 Oct;72(5): 570-5.

30 Shimura M, Yasuda K, Nakazawa T, Tamai M Visual dysfunction after panretinal photocoagulation in patients with severe diabetic retinopathy and good vision. Am J Ophthalmol. $2005 \mathrm{Jul}$; 140(1):8-15.

31 Luttrull JK, Musch DC, Spink CA. Subthreshold diode micropulse panretinal photocoagulation for proliferative diabetic retinopathy. Eye. 2008 May;22(5):607-12.

32 Kumar V, Ghosh B, Raina UK, Goel N. Subthreshold diode micropulse panretinal photocoagulation for proliferative diabetic retinopathy. Eye. 2009 Nov;23(11):2122-3.

33 The Early Treatment Diabetic Retinopathy Study Research Group. Fundus photographic risk factors for progression of diabetic retinopathy. ETDRS report number 12. Ophthalmology. 1991 May;98(5 Suppl 1):823-33.

34 Li Z, Song Y, Chen X, Chen Z, Ding Q. Biological modulation of mouse RPE cells in response to subthreshold diode micropulse laser treatment. Cell Biochem Biophys. 2015 Nov;73(2):545-52. 